\title{
Drowsiness Detection System
}

\author{
Suriya Kumar S, Kishor R, J.Kalaivani
}

\begin{abstract}
Melbourne is one of the liveliest cities in the world. It has a well efficient transport system, supported by a vast network of trams. Therefore, the mental health and stress level of the tram drivers plays a crucial role in the safety of the passengers. The issue of fatigue and drowsiness in the tram drivers are mostly due to their work-time and the most common thing is that the drowsiness occurs during the work time itself. This drowsiness is a risk for everyone including those who are not travelling in the tram. The current system that is used to prevent the drivers from falling sleeping is called the deadlock system. In this system the driver keeps his foot on a pedal at all times. Whenever the driver lifts his foot from the pedal the tram stops moving. Considering the technologies that are currently implemented in the vehicles seems to be insufficient. More over the driver gets uncomfortable when he keeps his foot onto the lever for a long time during long working hours. We have used OpenCV in python to create a program which monitors the eyes of a person and ensures that they keep the eyes open. The developed algorithm uses python libraries to detect any abnormality in the time interval between blinks and the extent of openness of the driver's eyes. When an abnormality is detected the driver receives an alarm on his phone indicating driver drowsiness.
\end{abstract}

Keywords : Micro-controllers ,Open CV, Raspberry Pi

\section{INTRODUCTION}

Drowsiness is a normal issue people face everyday. An average person requires 6-7 hours of sleep everyday. Drowsiness happens to occur due to various reasons like lack of sleep, stress, mental pressure and other similar human characteristics . In some cases, this may have a negative effect at the workplace. Few of which are jobs that require operation from the same place for a long time. The case we've considered here is the case of drivers. They are required to drive the vehicle continuously for longer hours. This might make the driver drowsy at times .

When this happens, there is a chance that the driver might doze off during the trip. This puts the travelers at risk and might result in an accident at times. To solve this problem, a safety device that can alert the driver when the driver is drowsy is created. This is done by mainly using a buzzer and a vibrating motor to alert the driver. This way the driver regains

Revised Manuscript Received on April 17, 2020.

* Correspondence Author

Kishor R*, Student, B.Tech, Computer Science Engineering course in SRM Institue of Science and Technology.

Suriya Kumar M, Student, B.Tech, Computer Science Engineering course in SRM Institue of Science and Technology

Dr.J.Kalaivani, Assistant Professor in the Department of Computer Science and Engineering from SRM Institute of Science and Technology

(c) The Authors. Published by Blue Eyes Intelligence Engineering and Sciences Publication (BEIESP). This is an open access article under the CC BY-NC-ND license (http://creativecommons.org/licenses/by-nc-nd/4.0/) control over the vehicle and is not drowsy anymore . Because its only normal that people feel drowsy from time to time. For this purpose , the traditional deadlock solution is used. This is done with the help of a Raspberry Pi 3 module and Open CV which uses Python and Scratch as programming languages.

\section{RELATED WORK}

Various methodologies have been made used of in detecting driver drowsiness. Motion sensors using SVM have been able to detect drowsiness level in drivers.For this purpose, raw motion data sets that include linear acceleration and radial velocity information were used. This enables the driver to have a distraction free drive. Ways to detect drowsiness using day time short nap has been achieved by using Bayesian non negative CP decomposition by determining CP rank. For this purpose, UCI data sets were used.

Another approach is to make use of Raspberry Pi 3.This is done by using a Raspberry Pi 3 module and camera using input from 10 volunteers.Flash JavaScript(FlashJaX) can also be made use of by implementing a cross platform.This can be achieved by using JavaScript and AdobeActionScript.A browser that supports cloning of the document node is created by FlashJaX and its given as input.Through this, Web security and protection from untrusted sites can be achieved.Mining behavior models from web applications using Process Crawler(ProCrawl) is used [10].A configured set of UI views are given as input for this case.This helps bring development, testing and maintenance to an easier level by removing any kind of complexity.Discovering vulnerabilities in the source code with fewer false positives [6] has also been successfully achieved using WAP. A large set of PHP applications are used for the input .This is done in order to reduce vulnerability in source codes written in unsafe languages.Facial expression recognition using RGB, 3D, Thermal and multi-modal approach has also been implemented successfully.

\section{MODULE DESCRIPTIONS}

\section{A. Hardware Components}

\section{- $\quad$ Raspberry Pi 2}

The Raspberry Pi 2 is a small sized board that can be used to explore computing.It can be connected to external devices like a monitor, TV, mouse or keyboard.Programming languages like Scratch and Python can be used to code. Our model uses Raspberry Pi 2 using Python. This code is mainly responsible for input and output from the

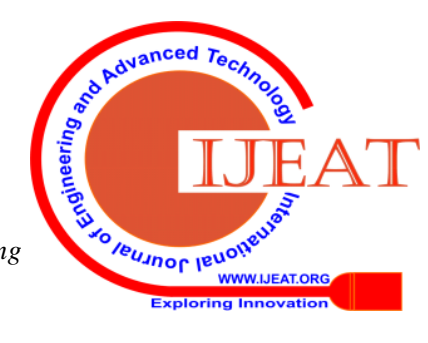


camera,measurement of facial features like the eye and the ear using $\mathrm{x}$ and $\mathrm{y}$ axes. It is also responsible to create a database of existing drivers and create new profile for new drivers.In case drowsiness is detected message will be sent to the owner.

\section{- GSM Module}

The GSM Module is used to hold a SIM card.Whenever drowsiness is detected a message from the SIM card mounted on the GSM Module is sent to the owner in addition to activation of the buzzer and the vibrator.For this to happen an app is to be installed by the owner.This app receives the message from the GSM Module.

The secondary hardware components used are camera, vibrator, buzzer, lithium ion battery. The camera is used to capture the drivers facial features and is used to recognize the driver.The vibrator vibrates when drowsiness is detected.The buzzer is also activated when drowsiness is detected and is used to alert the driver.The battery is used as a power source.

\section{B. Software Components}

\section{- Raspbian}

Raspbian is an operating system that is used to operate Raspberry Pi.It has various version like Raspbian Buster Raspbian Stretch.It is Linux based.It uses PIXEL, which is, Pi Improved X-Window Environment, Lightweight as its desktop environment.

\section{- Open CV}

Open CV stands for open source computer vision.It is basically a library of programming function.Its main aim is to solve computer vision to the related problem.In our case Open CV is mainly used to collect and store driver related information.It is mainly also used to detect the drivers face for any drowsiness and make changes in the database accordingly.

\section{WORKING METHODOLOGY}

The system is designed around Raspberry Pi (SBC). The system needs to be mounted in the front desk, In front of the car/vehicle driver. The system consists of SBC, Camera Module, Buzzer (Audio Alarm), Vibration motor and LCD module. The system operates in real time and captures the video of the drive and process it using SBC. Raspberry Pi (SBC) is used for the development of the system.

The Raspbian OS is installed onto the Raspberry pi SBC. Image processing performed using computer vision. The system automatically detects the face and eye and whether eye is open or closed. Once system detects the eye is closed for more time, it triggers an audio alarm and vibration alarm to notify the driver for drowsiness. Once the driver opens his eyes alarm stops automatically. The complete system runs onto $5 \mathrm{~V}$ power source.

A power source is used to power up the circuit elements .A camera module is used to capture the image of the driver which is sent as the initial input to the $\mathrm{Pi} 2$ micro-controller. Upon receiving the image the $\mathrm{Pi} 3$ processes the image to get the current status of the driver. To check the status of driver's eyes the eye threshold is defined in the program that is dumped on the micro-controller. The program is written using python language and the computer vision libraries are installed in the micro-controller module. When an abnormality in the driver's action are detected ,the buzzer is turned on while a vibrating alert is sent to the driver so that the driver can wake up.

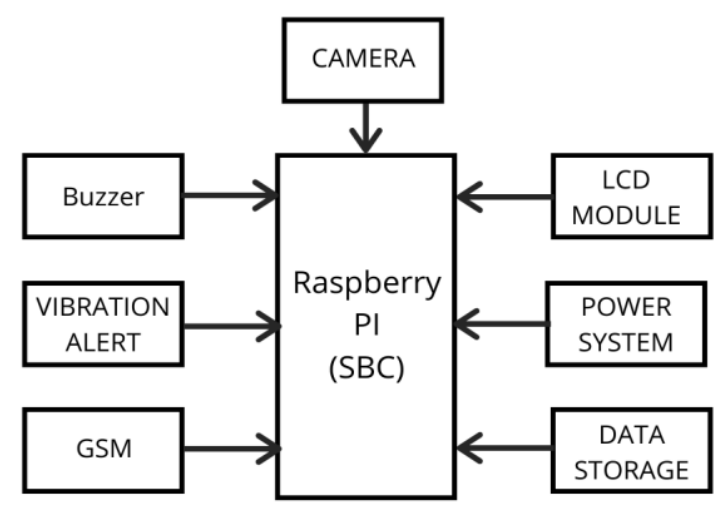

- Driver threshold details

The initial step is to have a database of all the drivers. So, this is done and in case a new face is detected, a new entry is made to the database. This includes information regarding the eye threshold and the ear. This information is stored in the form of graphs. When the camera detects any kind of drowsiness, it immediately recognizes the driver's face and makes a note of it.

\section{- Device connection}

When the driver is driving and starts to feel drowsy, the camera keeps monitoring the driver. Once the driver closes his eyes for more than a specific time, the buzzer starts buzzing and vibrator is activated. This alerts the driver and brings him back to a non drowsy state.

\section{- Message Transmission}

Another feature added to this model is the messaging feature.There is a central unit that controls the whole system as well. A person from the central unit must have an app installed in their phone. Once drowsiness is detected and the safety device is activated, a message is sent to the owner/central unit through the app. This is done with the help of the GSM module from which the message is sent.

\section{RESULTS}

The final output of our model is as follows :

1) Buzzer sound

2) Vibrating motor that produces vibrations

3) Messaging system

4) LCD module as a light source in the vehicle

\section{CONCLUSION}

The drowsiness detection model developed has proven to be very useful and is of extreme importance. A lot of people travel by public transport on a daily basis. These include buses, trams, taxi services ,etc. Hence it is of utmost importance to ensure the safety of these travelers

Published By:

Blue Eyes Intelligence Engineering

\& Sciences Publication

DOI: 10.35940/ijeat.D8489.049420

Journal Website: www.ijeat.org

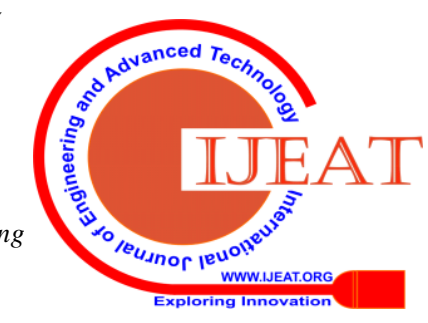


Our model, therefore, makes sure that drowsy drivers will not be a problem and hence the risk of accidents can be prevented .

Also, taking into consideration various factors like making changes to the speed of the vehicle or alerting other drivers around the vehicle or taking more precautionary measures can be considered for future enhancement to the existing ones.

\section{REFERENCES}

1. Ashish Naire, Vaijanath Patane, Umesh Patil, Prof. S.Y.Sawant, "Raspberry Pi based drowsiness detection", International Journal of Engineering Science and Computing System, 2018

2. Pranoto Hidaya Rusmin, Andrew B. Osmond, Arief Syaichu-Rohman, "Design and implementation of driver drowsiness detection system on Digitalized Driver System", IEEE 3rd International Conference on System Engineering and Technology, 2013

3. Javed Ahmed, Jian Ping-Li, Saeed Ahmed Kran, Riaz Ahmed Shakri, "Eye behavior based drowsiness detection system", School of Computer Science \& Engineering,2015

4. P.H.Phung,M.Monshizadeh,K.W.Hamlen,M.Sridhar,".Between Worlds:Securing Mixed JavaScript/ActionScript Multi-Party Web content",2015

5. B.-G. Lee, S.-J. Jung, W. -Y. Chung, "Realtime physiological and vision monitoring of vehicle driver for non-intrusive drowsiness detection", IET Commun., 2011

6. Iberia Medeiros,Nuno Neves,Miguel Correia."Detecting and removing Web applicationVulnerabilities with Static Analysis and Data Mining”,2016

7. Zhang X., Wang X., Yang X., Xu C., Zhu X., Wei J.”Driver drowsiness detection using mixed-effect ordered logit model considering time cumulative effect",Analytic Methods in Accident Research, Volume 26, 2020

8. Li J., Li H., Umer W., Wang H., Xing X., Zhao S., Hou J.,’Identification and classification of construction equipment operators' mental fatigue using wearable eye-tracking technology", Automation in Construction, Volume 109, 2020

9. Elamrani Abou Elassad Z., Mousannif H., Al Moatassime H., Karkouch A.,"The application of machine learning techniques for driving behavior analysis:" A conceptual framework and a systematic literature review Engineering Applications of Artificial Intelligence, Volume 87, 2020

10. M.Schur,A.Roth,A.Zeller."Mining workflow models from Web Applications",2015

\section{AUTHORS PROFILE}

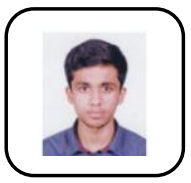

Kishor $\mathbf{R}$ is a Final Year B.Tech Student,pursuing Computer Science Engineering course inSRM Institue of Science and Technology.

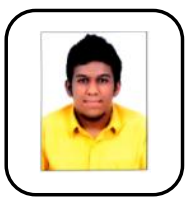

Suriya Kumar $\mathbf{M}$ pis a Final Year B.Tech Student,pursuing Computer Science Engineering course inSRM Institue of Science and Technology.

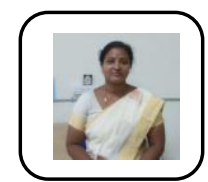

Dr.J.Kalaivani is an Assistant Professor in the Department of Computer Science and Engineering from SRM Institute of Science and Technology, whose specialization is in Computer networks.

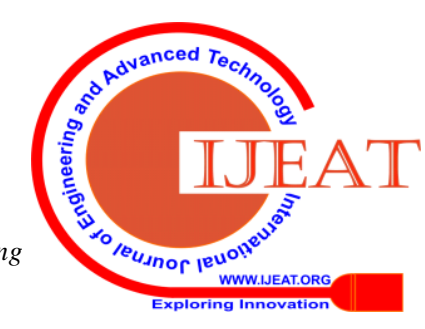

